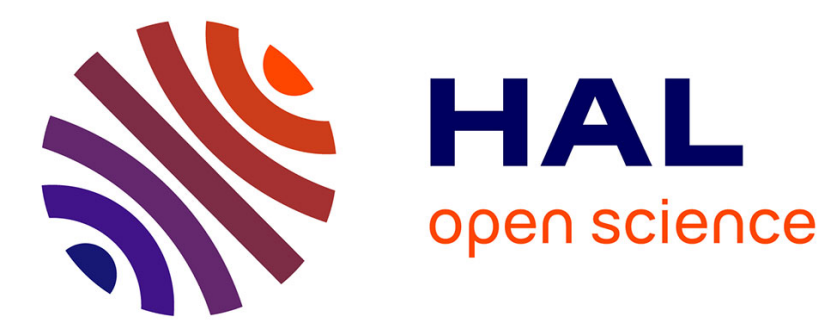

\title{
Efficient stochastic simulation approach for RTM process with random fibrous permeability
}

F. Zhang, B. Cosson, S. Comas-Cardona, C. Binetruy

\section{To cite this version:}

F. Zhang, B. Cosson, S. Comas-Cardona, C. Binetruy. Efficient stochastic simulation approach for RTM process with random fibrous permeability. Composites Science and Technology, 2011, 71 (12), pp.1478. 10.1016/j.compscitech.2011.06.006 . hal-00786583

\section{HAL Id: hal-00786583 \\ https://hal.science/hal-00786583}

Submitted on 9 Feb 2013

HAL is a multi-disciplinary open access archive for the deposit and dissemination of scientific research documents, whether they are published or not. The documents may come from teaching and research institutions in France or abroad, or from public or private research centers.
L'archive ouverte pluridisciplinaire $\mathbf{H A L}$, est destinée au dépôt et à la diffusion de documents scientifiques de niveau recherche, publiés ou non, émanant des établissements d'enseignement et de recherche français ou étrangers, des laboratoires publics ou privés. 


\section{Accepted Manuscript}

Efficient stochastic simulation approach for RTM process with random fibrous permeability

F. Zhang, B. Cosson, S. Comas-Cardona, C. Binetruy

PII:

S0266-3538(11)00211-9

DOI:

10.1016/j.compscitech.2011.06.006

Reference:

CSTE 5005

To appear in:

Composites Science and Technology

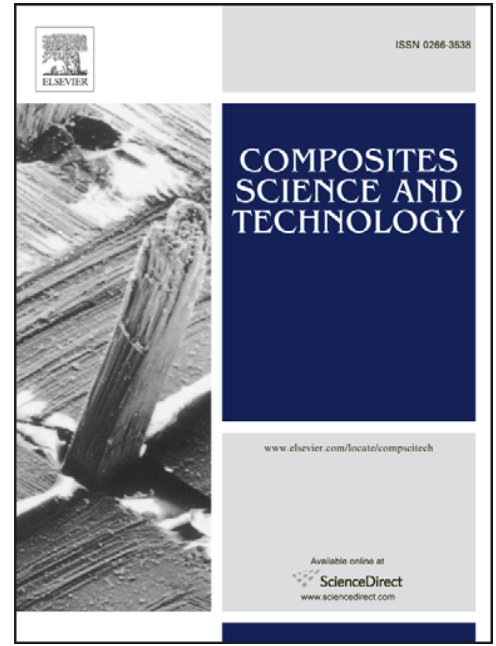

Received Date: $\quad 28$ December 2010

Revised Date: $\quad 13$ June 2011

Accepted Date: $\quad 14$ June 2011

Please cite this article as: Zhang, F., Cosson, B., Comas-Cardona, S., Binetruy, C., Efficient stochastic simulation approach for RTM process with random fibrous permeability, Composites Science and Technology (2011), doi: 10.1016/j.compscitech.2011.06.006

This is a PDF file of an unedited manuscript that has been accepted for publication. As a service to our customers we are providing this early version of the manuscript. The manuscript will undergo copyediting, typesetting, and review of the resulting proof before it is published in its final form. Please note that during the production process errors may be discovered which could affect the content, and all legal disclaimers that apply to the journal pertain. 


\title{
Efficient stochastic simulation approach for RTM process with random fibrous permeability
}

\author{
F. Zhang, B. Cosson, S. Comas-Cardona, C. Binetruy* \\ Ecole des Mines de Douai, Polymers and Composites Technology \& Mechanical \\ Engineering Department, 941 Rue Charles Bourseul, 59508 Douai Cedex, France
}

\begin{abstract}
An efficient numerical approach is presented for the simulation of mold filling process with random fibrous permeability as input. Suitable dimension-reduction techniques are employed to represent all the random fields in the physical system. The required accuracy in tracking the moving resin flow front is guaranteed by the Level Set Method (LSM). The Probabilistic Collocation Method (PCM) is applied in the efficient solution of the non-linear stochastic system. Numerical examples for different injection schemes are presented to demonstrate the cost-effectiveness of the current approach in predicting the variability in mold filling results in comparison to the traditional Monte Carlo Simulation (MCS).
\end{abstract}

Keywords: B. Permeability; C. Stochastic simulation; C. Random field; C. Level set method (LSM); E. Mold filling process.

* Corresponding author. Tel. +33 (0)3 27712175

christophe.binetruy@mines-douai.fr (Christophe Binetruy) 


\section{Introduction}

The resin transfer molding (RTM) technique, consisting of the impregnation of a fibrous preform by a reactive resin and the curing of the part, is widely applied in advanced composites manufacturing. Irregular flow patterns or defects in the final products often occur due to the high variability in the transport properties of the fibrous media. Therefore, RTM robustness has to be studied to avoid trial and error procedures.

Experimental studies [1-4] show the scatter in measured global permeability data can be up to 0.5 in terms of coefficient of variation from batch to batch of the same material. Besides, local permeability exhibits spatially-dependent fluctuation due to its inherently heterogeneous architecture. Although studies have been performed on the probabilistic characterization of the random transport properties of fibrous media [2-4], the random effects are usually neglected in the available optimization or on-line control schemes [5-7] which employ common simulation softwares for mold filling. Available stochastic studies in the RTM context [8-12] mostly depend on the Monte Carlo simulation (MCS), which is computationally expensive for a fast uncertainty analysis or to be combined with other algorithms for optimization or sensitivity analysis.

In this paper, an efficient stochastic simulation tool for mold filling process is developed, which is able to accurately predict the statistics of responses at a fraction of the cost of the MCS. The approach is implemented based on available numerical techniques in the stochastic and flow simulation domain, which are explained in details in Section 2. Numerical examples are given in Section 3 to illustrate the cost-effectiveness of the current method in uncertainty quantification, using the MCS as reference. Main conclusions and future outlook are discussed in Section 4.

\section{Stochastic simulation approach for mold filling process}

\subsection{Dimension reduction for the stochastic system}


As fiber reinforcement permeability is function of local architectural parameters such as fiber volume fraction and fiber tow orientation $[3,4,10,12]$, it can be described by a random field $K(x, \omega)$ with $\omega$ as a variable in the random space. For this application, $K(x, \omega)$ is simplified as a weak stationary random field with mean $\left(\mu_{K}\right)$ and autocovariance independent of the absolute location, i.e.

$$
\begin{gathered}
E[K(x, \omega)]=\mu_{K} \\
C_{K K}[\Delta x]=\operatorname{Cov}[K(x, \omega) K(x+\Delta x, \omega)]=E\left[\left(K(x, \omega)-\mu_{K}\right)\left(K(x+\Delta x, \omega)-\mu_{K}\right)\right]
\end{gathered}
$$

The reliable data on the marginal Probability Density Function (PDF) of fiber permeability is very limited. Some studies [2] presumed a Gaussian distribution for the measured global permeability data, while the log-normal distribution is adopted for $K(x, \omega)$ in this study, in order to ensure the nonnegativeness of permeability data even in case of a high variance.

In analogy with the discretization of a deterministic property field for numerical simulation, the random field $K(x, \omega)$ has to be discretized in both spatial and probabilistic spaces to reduce the dimensions of the corresponding stochastic system and to characterize it by the Stochastic Partial Differential Equations (SPDEs). Focusing on the underlying Gaussian field $Y(x, \omega)=\log (K(x, \omega))$, its discretization can be performed using the Karhunen-Loève expansion (KLE) [13], by projecting $Y(x, \omega)$ onto a set of uncorrelated random basis functions with corresponding deterministic coefficients, i.e.

$$
Y(x, \omega)=\mu_{Y}+\sum_{i=1}^{M} \sqrt{\lambda_{i}} \phi_{i}(x) \xi_{i}(\omega)
$$

In Eq. (2), M is the order of the KLE which controls the accuracy of the expansion. It has been proved that the random basis $\left\{\xi_{i}(\omega)\right\}(\mathrm{i}=1,2, \ldots, \mathrm{M})$ is a collection of 
independent standard Gaussian random variables for a Gaussian random field [13]. The corresponding deterministic coefficients $Y_{i}(x)=\sqrt{\lambda_{i}} \phi_{i}(x)$ are determined by solving Eq. (3) for the eigenvalues $\left\{\lambda_{i}\right\}$ and eigenfunctions $\left\{\phi_{i}(x)\right\}$ of the covariance of $Y(x, \omega)$ (denoted by $C_{Y}\left(x_{1}, x_{2}\right)$ ).

$$
\int_{D} C_{Y}\left(x_{1}, y_{1} ; x_{2}, y_{2}\right) \phi_{i}\left(x_{2}, y_{2}\right) d x_{2} d y_{2}=\lambda_{i} \phi_{i}\left(x_{1}, y_{1}\right)
$$

In order to reduce the computational effort in solving Eq. (3), the eigenvalues and eigenfunctions are assumed in the form of $\lambda_{i}=\lambda_{i}^{(1)} \lambda_{i}^{(2)}, \phi_{k}(x, y)=\phi_{i}^{(1)}(x) \phi_{i}^{(2)}(y)$, and the covariance is chosen as a parametric model separable in two directions:

$$
C_{Y}\left(x_{1}, y_{1} ; x_{2}, y_{2}\right)=\sigma_{Y}^{2} \exp \left(-\frac{\left|x_{1}-x_{2}\right|}{l_{x}}-\frac{\left|y_{1}-y_{2}\right|}{l_{y}}\right)
$$

with the correlation length $l_{x}, l_{y}$ (in $\mathrm{x}$, y direction) depending on the fabric architecture. Using the architectural parameters of reinforcement (e.g. inter-tow gap width for directional fabrics, porosity for random mat, etc.) acquired from image analysis or other measurements, realizations of permeability field can be obtained by means of numerical or analytical methods based on representative unit cell of the fabric structure. Then, optimization algorithms $[11,12]$ can be applied to fit the experimental realizations into Eq. (4) to estimate $l_{x}, l_{y}$. While this procedure is under progress, a trial value for the correlation length is used in the numerical examples.

Once Eq. (3) is reduced to a set of 1-D equations for each direction, it can be transformed by means of the Galerkin method into generalized eigenvalue problems, from which the set $\left\{\lambda_{i}\right\}$ and $\left\{\phi_{i}(x)\right\}$ are solved numerically. The log-normal random field is accordingly represented in terms of discrete random variables:

$$
K(x, \omega)=\exp \left(\mu_{Y}+\sum_{i=1}^{M} \sqrt{\lambda_{i}} \phi_{i}(x) \xi_{i}(\omega)\right)
$$


As the random dimension of the input of the stochastic system is reduced to M, any random output, as non-linear function of the input, should be represented by the same random basis $\left\{\xi_{i}\right\}$. Without knowing the marginal PDF, the Polynomial Chaos Expansion (PCE) [13] is employed to represent various outputs. For example, the pressure field is expressed in the PCE as

$$
p(x, \omega)=\sum_{j=0}^{P} p_{j}(x) \psi_{j}(\xi(\omega))
$$

where $\left\{\psi_{j}(\xi)\right\}(\mathrm{j}=0,1, \ldots, \mathrm{P})$ are M-dimensional Hermite polynomials in terms of $\xi=\left\{\xi_{i}\right\} \quad(\mathrm{i}=1,2, \ldots, \mathrm{M})$ following the joint Gaussian PDF [13], and $p_{j}(x)$ denotes unknown deterministic coefficients. The order of the PCE (p) is taken as the highest order of the Hermite polynomials, related to the number of terms by $P+1=(p+M) ! / p ! M$ ! [13]. Once the deterministic coefficients are solved, the statistics of the target random response can be easily obtained by performing the MCS on its PCE.

\subsection{Stochastic model for mold filling process}

As a first step, only permeability uncertainty and the isothermal processes are considered (other random inputs or process models are ready to be incorporated in the presented stochastic framework). Following the well-established deterministic simulation models for RTM process $[9,14]$, resin flow through fibrous media is approximated by a quasi-steady process. Each intermediate state is governed by Darcy's law and the continuity equation of an incompressible fluid with relevant random quantities represented by the suitable expansions:

$$
\nabla \cdot\left[\exp \left(\mu_{Y}+\sum_{i=1}^{M} \sqrt{\lambda_{i}} \phi_{i}(x) \xi_{i}\right) \cdot \sum_{j=0}^{P} \nabla p_{j}(x) \psi_{j}(\xi)\right]=Q(x)
$$

where $Q(x)$ is the source term (which can also be random if needed). In order to simulate the moving resin flow front, most commercial codes employ the Control 
Volume / Finite Element Method (CV/FEM) [14], in which the flow front position is represented by the filling fraction in the control volumes. Due to the dependence of the flow front shape on the discretization of the computational domain, it is not efficient in simulating distinct and accurate flow fronts, which are required for the current study. As an alternative, the Level Set Method (LSM) [15] motivated by the multi-phase flow problems [16] begins to be adopted for LCM simulation $[17,18]$. The basic idea of this technique is to represent the moving interface by a continuous function $\phi(x)$ (i.e. level set function) taking opposite signs in different media (impregnated or dry) and zero on their interface (resin-air flow front). In the current context, the level set function is a random variable $\phi(x, \omega)$ representing the signed distance from location $\mathrm{x}$ to the flow front with a random location. Following the classical LSM [15], the movement of resin flow front during a certain time step is characterized using the advection equation [15]

$$
\frac{\partial \phi(x, \omega)}{\partial t}+u(x, \omega) \cdot \nabla \phi(x, \omega)=0
$$

with the Darcy velocity derived from the expansions of permeability and pressure

$$
u(x, \omega)=-\frac{1}{\mu} \exp \left(\mu_{Y}+\sum_{i=1}^{M} \sqrt{\lambda_{i}} \phi_{i}(x) \xi_{i}(\omega)\right) \cdot \sum_{j=0}^{P} \nabla p_{j}(x) \psi_{j}\left(\xi_{i}(\omega)\right)
$$

Besides, the signed distance feature of the level set function can only be preserved by performing reinitialization (Eq. 10) at certain time intervals.

$$
\left\{\begin{array}{c}
\frac{\partial \varphi}{\partial \tau}+\operatorname{sign}(\phi(x, \omega))(|\nabla \varphi|-1)=0 \\
\varphi(x, 0)=\phi(x, \omega)
\end{array}\right.
$$

where $\tau$ denotes the artificial time variable. $\phi(x, \omega)$ is then replaced by the solution $\varphi(x)$ (also random actually) which is a signed distance function with the same zero-contour, i.e. flow front. The details of numerical solution for Eq. (8) and (10) can be referred to $[15,16]$. 


\subsection{Solution of the stochastic model}

Before choosing a suitable solution technique for Eqs. (7) (10), it is noted that, for the stochastic modeling of RTM process, a particular difficulty consists in the characterization of the random resin/air interface in the computation domain, represented by the zero-level set. Due to the signed distance requirement for any valid realization of a random level set function $\phi(x, \omega)$, it is impossible to assume $\phi(x, \omega)$ as a stationary random field. For this reason, application of the random level set function has only been tempted for the random boundaries with simple geometries [19], which is far from the current case of an irregular interface. However, the uncertainty propagation in the model necessitates the randomness in the flow front or the level set function. Therefore, a stochastic solution technique able to circumvent the direct representation of $\phi(x, \omega)$, i.e. a so-called non-intrusive stochastic method [20] which deals with the whole non-linear system of equations as a black-box, is needed.

The Probabilistic Collocation Method (PCM) [20,21] is found to be an optimal choice. Focusing on an interesting random output of the model, e.g. the fill time $T(x, \xi)$, the stochastic system (Eqs. (7) (10)) can be represented by an implicit function

$$
T(x, \xi)=f\left(\exp \left(\mu_{Y}+\sum_{i=1}^{M} \sqrt{\lambda_{i}} \phi_{i}(x) \xi_{i}\right)\right)
$$

As mentioned in Section 2.1, the random output can be approximated by the PCE:

$$
T(x, \xi) \approx \sum_{j=0}^{P} T_{j}(x) \psi_{j}(\xi)
$$

Thus, the solution is interpreted as seeking the set of unknown coefficients $\left\{T_{j}(x)\right\}$ to minimize the residual of Eq. (12) in the statistical sense. Following the notion of weighted residual method, the mean residual can be expressed as 


$$
\int_{\xi}\left(T(x, \xi)-\sum_{j=0}^{P} T_{j}(x) \psi_{j}(\xi)\right) w_{k}(\xi) p(\xi) d \xi=0
$$

where $w_{k}(\xi)$ are weight functions to choose and $p(\xi)$ the joint PDF of the random vector $\xi$. Starting from Eq. (13), different choice of $w_{k}(\xi)$ will lead to different stochastic solution technique. For the instance of the PCM, the zero residual is enforced at a set of collocation points $\left\{\xi_{k}\right\}(\mathrm{k}=1,2, \ldots, \mathrm{N})$ in sample space (equivalent to using the Dirac- $\delta$ functions at the collocation points as weight functions $w_{k}(\xi)=\delta\left(\xi-\xi_{k}\right)$ ). The integration is then reduced to a linear system of equations of the unknown coefficients $\left\{T_{j}(x)\right\}$

$$
\sum_{j=0}^{P} T_{j}(x) \psi_{j}\left(\xi_{k}\right)=T\left(x, \xi_{k}\right)
$$

with the coefficient matrix $\psi_{j}\left(\xi_{k}\right)$ and the right-hand-side $T\left(x, \xi_{k}\right)$ evaluated at $\left\{\xi_{k}\right\}$ by means of the PCE and the deterministic system of equations, respectively. Therefore, the computational effort for solving the stochastic system is reduced to that of solving the deterministic system for $\mathrm{N}$ times, which means the PCM has great advantage in terms of the efficiency and convenience in operation.

However, the accuracy in predicting the response statistics is the primary factor in assessing the performance of the PCM. Since the zero residual is only ensured at the set of collocation points, they must be able to capture the high probability region in the sample space. In analogous to the Gaussian quadrature, the collocation points are usually chosen from the roots of the polynomials of one order higher than the PCE, and kept as close to the origin as possible in sample space [20,21]. For example, if the $2^{\text {nd }}$-order PCM is employed, the optimal collocation points should have the coordinates (in sample space) 0 or $\pm \sqrt{3}$ (as roots of $H_{3}(\xi)=\xi^{3}-3 \xi$ ). 
Up to now, the overall framework (implemented in MATLAB programming language) for uncertainty quantification in the RTM process model is completed based on the PCM and the LSM, as illustrated in the flowchart in figure 1.

\section{Numerical examples}

The effectiveness of the approach developed above for the uncertainty analysis is demonstrated by applying it to a simple two-dimensional mold filling model. The mold has a thickness of 5mm, a single inlet/outlet and an internal insert (figure 2(a)), containing an isotropic fabric of $30 \%$ fiber volume fraction. The fibrous permeability, assumed as a stationary log-normal random field with the mean value $\mathrm{K}_{0}=1 \times 10^{-9} \mathrm{~m}^{2}$, a coefficient of deviation $\mathrm{CV}=1.0$ (to illustrate the robustness of the method) and the covariance function described by Eq. (4) with a correlation length equaling half the mold size, is taken as the only source of input uncertainty of the system. The $4^{\text {th }}$-order $\mathrm{K}-\mathrm{L}$ expansion is performed on the permeability field, from which the generated realizations can reproduce the input statistics (e.g. the local PDF and the autocorrelation) with acceptable accuracy, as shown in figures 2 (a) (b). Resin with viscosity of $0.1 \mathrm{~Pa} \cdot \mathrm{s}$ is injected into the mold by means of two different schemes respectively. In each case, the deterministic solution resulting from a homogeneous fabric with permeability $\mathrm{K}_{0}$ is used to normalize the mean of the stochastic solution to highlight their discrepancy. The stochastic simulation is performed (on a CPU of Intel Xeon X5550/2.67GHz) using the $2^{\text {nd }}$-order PCM (with 15 collocation points selected following the rules in Section 2.3) and also the MCS with $10^{3}$ realizations for validation.

\subsection{Constant flow rate driven injection}

First, the injection case using a constant flow rate $\left(5 \times 10^{-6} \mathrm{~m}^{3} / \mathrm{s}\right)$ is considered. In order to design the appropriate stiffness and closing force of the mold for the targeted thickness or fiber volume fraction [22, 23], the range of resin pressure magnitude needs 
to be known. However, without taking into account the uncertainty in pressure field, the maximal pressure magnitude may be underestimated by traditional simulation codes, which increases the risk of preform deformation and mold deflection.

The capability of the current method is first validated by the converged solution of the MCS, taking the peak value of the inlet pressure as the random response of interest. As shown in figure 3(a), the converged mean value can be taken as obtained by the MCS with more than 300 realizations, while the PCM only needs a cost of $5 \%$ of the former to get the same result with negligible error. To get more detailed probabilistic information, the Cumulative Density Function (CDF) of the random response can be employed. Figure 3(b) compares the CDF estimated by the $2^{\text {nd }}$-order PCM to those by the MCS with $10^{2}$ or $10^{3}$ realizations, showing that the convergence of the overall CDF can be accurately reached by the PCM (except the tail region of $\mathrm{CDF}>0.87$ ) at a cost less than $3 \%$ of that of the MCS.

Using the convergent stochastic solution, the evolution of the mean and the CV of the inlet pressure during the injection process are displayed in figures 4(a) (b), respectively, showing the consistency of the PCM estimation with the MCS standard through the time history. Results show that the long-term response (i.e. the average response of a large sample) is underestimated by the deterministic simulation, and the discrepancy may be significant for an input with high variance, as figure 4(b) shows that the CV of the response (around 0.65 0.70) is comparable to that of the input. Besides, the PCM prediction of the $\mathrm{CV}$ of inlet pressure increases as flow front advances, demonstrating the uncertainty propagation in the model. In addition, the peak in the mean value between 300 and $350 \mathrm{~s}$ with a magnitude comparable to the maximal value reflects the disturbance of the internal insert (referring to the isochrones in figure 2(a)). Due to the simple configuration of the mold, the spatial distribution of the mean and CV of the 
random pressure field are both analogous to that of the deterministic pressure field and not displayed here. Results show that the $\mathrm{CV}$ of pressure at any location (except those close to outlet) is largely in the range of $0.6 \sim 0.7$, which confirms the importance of the pressure variability for mold design.

The irregular flow pattern resulting from the random permeability field is the main obstacle for mold design. As a basis of the optimization schemes, the simulation tool should be able to assess the impact of the random input on the flow pattern. The resin-arriving time (referred to as fill time below) at any location is taken as the random variable quantifying the uncertainty in flow front. First, the PCM prediction for the mean and $\mathrm{CV}$ of fill time is compared to the respective MCS solution and validated (both have a slight dependence on discretization but only at a local scale). As can be seen in figures $5(\mathrm{a})(\mathrm{b})$, the mean value has small fluctuation around the deterministic one, implying a minor fluctuation in the flow pattern. Figures $5(\mathrm{c})(\mathrm{d})$ show that the $\mathrm{CV}$ of the fill time has a maximum of around 0.15 , with relatively higher values in the regions close to the corners or affected by the insert. However, the uncertainty in permeability field has no major influence on the flow pattern for this injection scheme.

\subsection{Constant pressure driven injection}

As an alternative, the constant pressure injection is widely adopted in practice because of its low cost of injection device and the steady pressure level. However, the total fill time has to be controlled respecting the resin reactivity and process automation. Some techniques aiming at the prediction of accurate fill time are tempted [24], but none focus on the random effects. Using a test example with inlet pressure given as $0.2 \mathrm{MPa}$, the influence of the input uncertainty on the statistics of the fill time is analyzed by means of the current approach.

In analogy to the precedent injection case, the convergence for the typical statistics of 
the mold fill time is first studied. For the mean value, the MCS requires at least 600 realizations to obtain the steady value, which can be predicted by the $2^{\text {nd }}$-order PCM with a relative error less than $2 \%$ using about $2.5 \%$ computational effort (figure 6(a)). Comparing the $\mathrm{CDF}$ curves in figure 6(b), the PCM result can match the overall shape and capture the tail region of the MCS estimation using $10^{3}$ realizations (approximately taken as converged).

In addition to the overall fill time for the mold, the randomness in the flow pattern can be easily investigated using the current method. Still using the converged MCS solution for validation, the mean value of the local fill time (normalized by the deterministic value) is displayed in figures 7(a)(b) for both methods. Simulation results show that at any location, the mean of random fill time is longer than the deterministic prediction by about $15 \%$ 30\%. The two methods give similar prediction in terms of magnitude and spatial distribution. The discrepancy between the stochastic and the deterministic solutions demonstrates the importance of the random effects for choosing suitable process parameters to account for the variation in the material properties.

The uncertainty propagation can be observed from the CV of the fill time (figure 7(c)(d)) which increases as the flow front moving forward, reaching about 0.8 (comparable to the $\mathrm{CV}$ of the input random field) near the outlet. Comparing to the results of constant flow rate injection (figure 5), the constant pressure injection appears more sensitive to the input uncertainty, in terms of the variability in the flow pattern and in mold fill time. By comparison to the MCS ( $10^{3}$ realizations) estimation, the PCM is able to predict accurately the statistical moments of the fill time which is an implicit function of the permeability, and its capability of randomness propagation is proved.

\section{Conclusions}

A stochastic simulation code has been developed by combining the Level Set technique 
with the Probabilistic Collocation Method, taking the advantage of efficiency in both the moving interface simulation and uncertainty propagation, and thus suitable to be embedded as a module into optimization or sensitivity analysis algorithms [25], instead of the time-consuming MCS. Numerical examples illustrate the capability of the developed simulation code in uncertainty quantification in the mold filling problem, as well as its great advantage over the MCS in terms of computational efficiency (e.g. for the given numerical tests, the CPU cost of the PCM is on average less than $5 \%$ of that of the MCS) for predicting the response statistics with comparable accuracy. Stochastic simulation results of simple mold filling processes reveal distinct discrepancies between the stochastic and deterministic solutions for various responses, which indicates the necessity to take into account the intrinsic randomness in transport properties.

In spite of the simple numerical tests shown in this paper, potential application in many aspects can be developed based on the current framework, such as the simulation of RTM process with multiple sources of input uncertainty (e.g. the race-tracking permeability, defects distributed in fabric, randomness in resin viscosity and curing), or the optimization of process parameters to reduce the sensitivity of outputs to the input uncertainty.

Acknowledgements: The authors would like to acknowledge the Conseil Régional du Nord - Pas de Calais (France) for the financial support for this study.

\section{References}

[1] Lundström TS, Stenberg R et al. In-plane permeability measurements: a nordic round-robin study. Composites: Part A 2000; 31: 29-43.

[2] Hoes K, Dinescu D, Sol H, Vanheule M et al. New set-up for measurement of permeability properties of fibrous reinforcements for RTM. Compos. Part A 2002; 33: 959-69.

[3] Desplentere F, Lomov SV et al. Micro-CT characterization of variability in 3D textile 
architecture. Composites Science and Technology 2005; 65: 1920-30.

[4] Endruweit A, McGregor P, Long AC et al. Influence of the fabric architecture on the variations in experimentally determined in-plane permeability values. Composites Science and Technology 2006; 66: 1778-92.

[5] Hsiao KT, Advani SG. Flow sensing and control strategies to address race-tracking disturbances in RTM. Part I: design and algorithm development. Composites: Part A 2004; 35: 1149-59.

[6] Nielsen DR, Pitchumani R. Closed-loop flow control in RTM using real-time numerical process simulations. Composites Science and Technology 2002; 62: 283-98.

[7] Lee DH, Lee WI, Kang MK. Analysis and minimization of void formation during resin transfer molding process. Composites Science and Technology 2006; 66: 3281-89.

[8] Markicevic B, Heider D, Advani SG et al. Stochastic modeling of preform heterogeneity to address dry spots formation in the VARTM Process. Composites: Part A 2005; 36: 851-58.

[9] Padmanabhan SK, Pitchumani R. Stochastic modeling of nonisothermal flow during resin transfer molding. International Journal of Heat and Mass Transfer 1999; 42: 3057-70.

[10] Verleye B, Lomov SV, Long A et al. Permeability prediction for the meso-macro coupling in the simulation of the impregnation stage of RTM. Composites: Part A 2010; 41: 29-35.

[11] Li J, Zhang C, Liang $\mathrm{R}$ et al. Statistical characterization and robust design of RTM processes. Composites: Part A, 2005; 36: 564-80.

[12] Skordos AA, Sutcliffe MPF. Stochastic simulation of woven composites forming. Composites Science and Technology 2008; 68: 283-96.

[13] Ghanem RG, Spanos S. Stochastic finite elements: a spectral approach (revised edition). Dover Publications, Inc., 2003.

[14] Lam YC, Joshi SC, Liu XL. Numerical simulation of the mould-filling process in resin-transfer moulding. Composites Science and Technology, 2000; 60: 845-55.

[15] Osher S, Fedkiw R. Level set methods and dynamic implicit surfaces. Springer-Verlag New York, Inc., 2003.

[16] Tornberg A-K, Engquist B. A finite element based level set method for multiphase flow. Computing and Visualization in Science, 2000; 3: 93-101.

[17] Soukane S, Trochu F. Application of the level set method to the simulation of resin transfer molding. Composites Science and Technology, 2006; 66: 1067-80.

[18] Gantois R, Cantarel A, Dusserre G et al. Numerical simulation of resin transfer molding using BEM and level set method. Int J Mater Form. 2010; 3 Suppl 1: 635-38.

[19] Nouy A, Clement A, Schoefs F et al. An extended stochastic finite element method for solving stochastic partial differential equations on random domains. Comput. Methods Appl. Mech. Engrg. 2008; 197: 4663-82.

[20] Stefanou G. The stochastic finite element method: Past, present and future. Comput. Methods Appl. Mech. Engrg. 2009; 198: 1031-51. 
[21] Huang S, Mahadevan S, Rebba R. Collocation-based stochastic finite element analysis for random field problems. Probabilistic Engineering Mechanics, 2007; 22: 194-205.

[22] Comas-Cardona S, Groenenboom P, Binetruy C et al. A generic mixed FE-SPH method to address hydro-mechanical coupling in LCM processes. Composites: Part A, 2005; 36: 1004-10. [23] Walbran W, Bickerton S, Kelly P. Measurements of normal stress distributions experienced by rigid liquid composite moulding tools. Composites: Part A, 2009; 40: 1119-33.

[24] Deleglise M, Binetruy C, Krawczak P. Solution to filling time prediction issues for constant pressure driven injection in RTM. Composites: Part A, 2005; 36 : 339-44.

[25] Sudret B, Der Kiureghian A. Comparison of finite element reliability methods. Probability Engineering Mechanics. 2002; 17: 337-48.

\section{Figures}

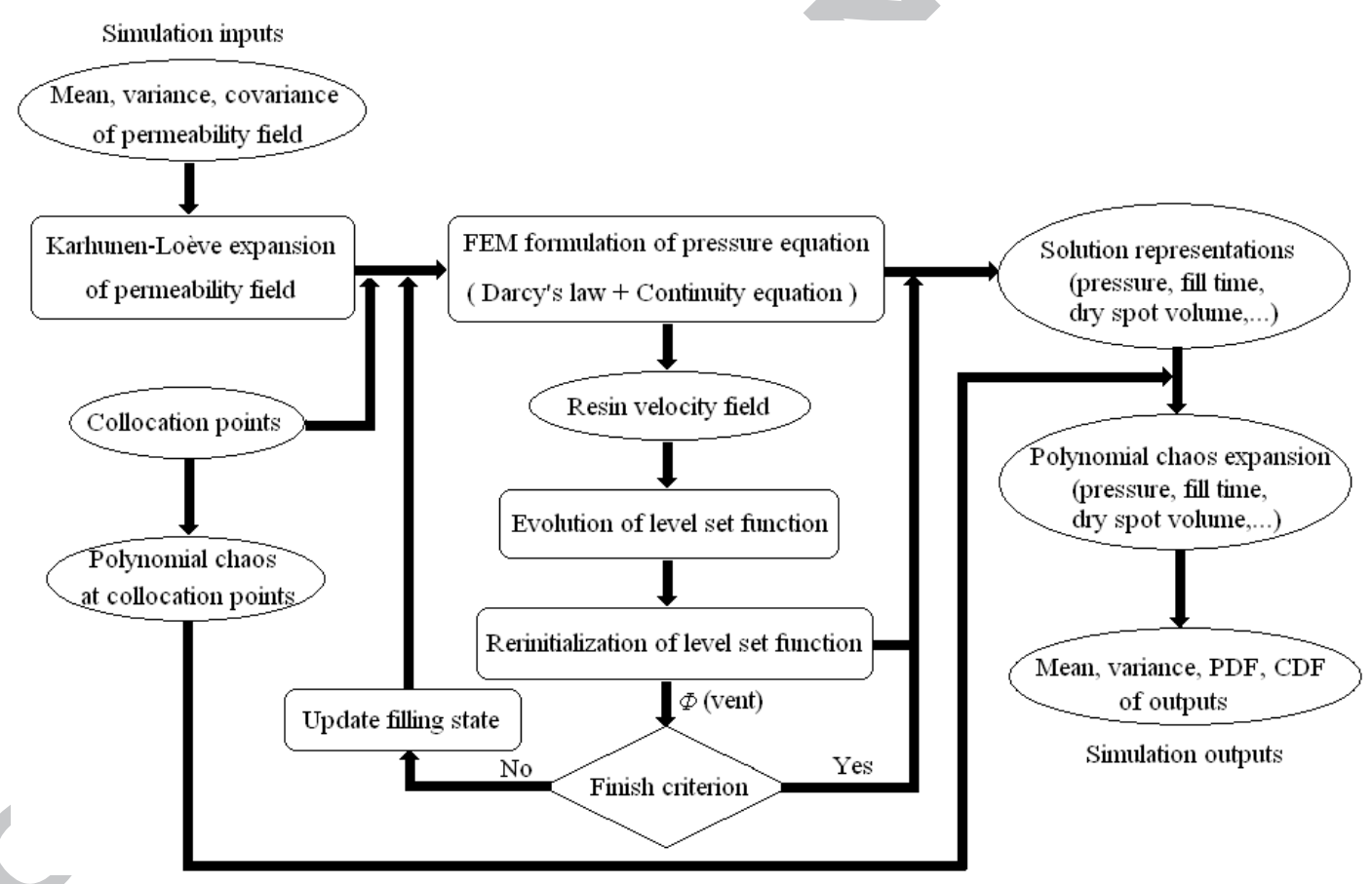

Fig. 1 Flowchart of simulation procedure 


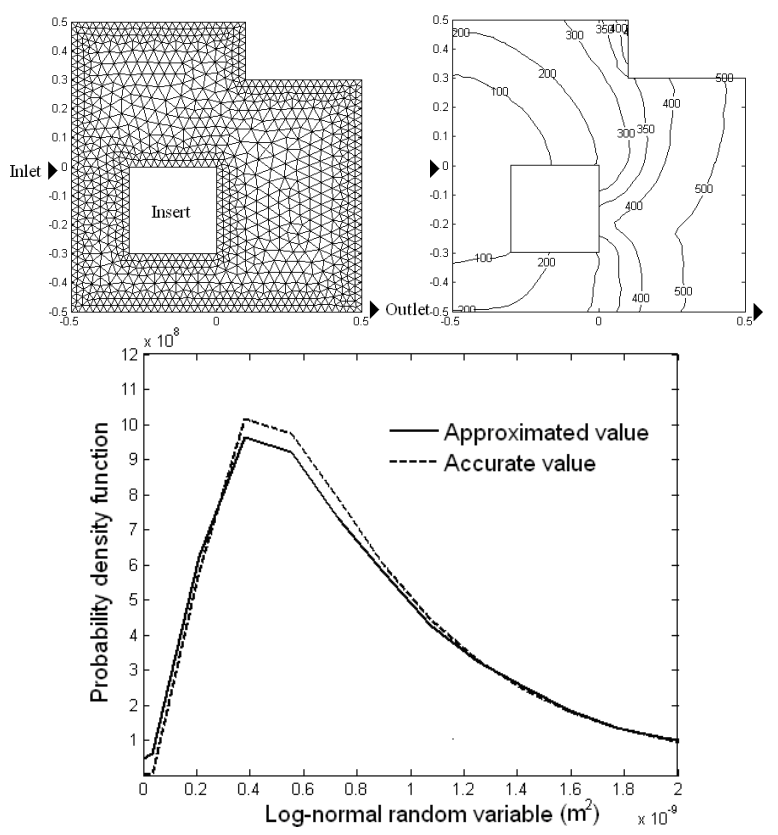

(a)
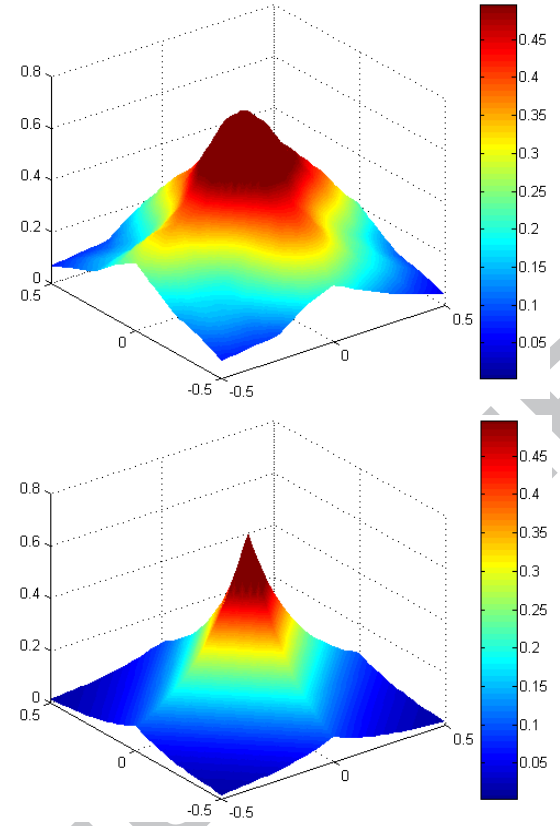

(b)

Fig. 2 Computational domain and K-L approximation of input statistics:

(a) upper-left: computational domain (discretized by 2010 triangular elements); upper-right: deterministic flow fronts at time 100, 200, 300, 350, 400, 500 (s); lower: local PDF (comparing simulated and accurate values);

(b) autocorrelation function with respect to location $(0,0)$ :

upper: simulated values; lower: analytical values.

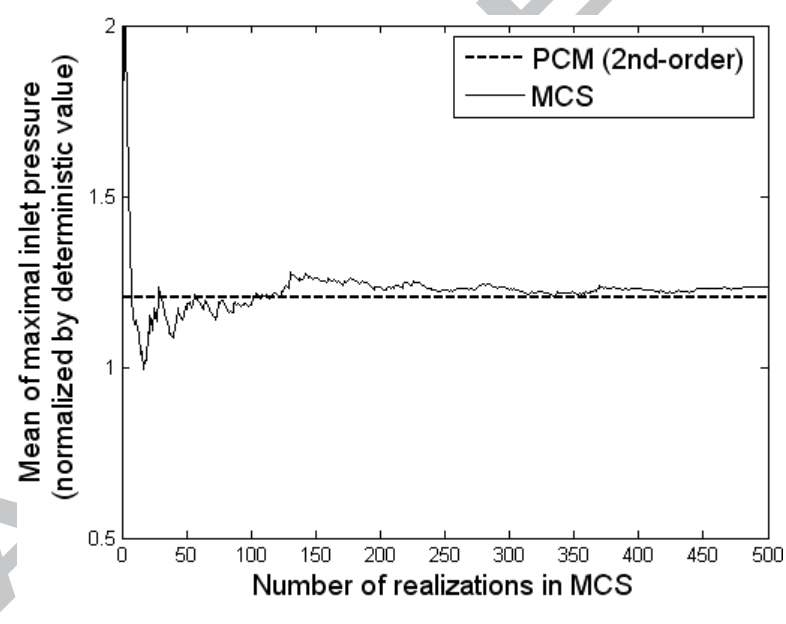

(a)

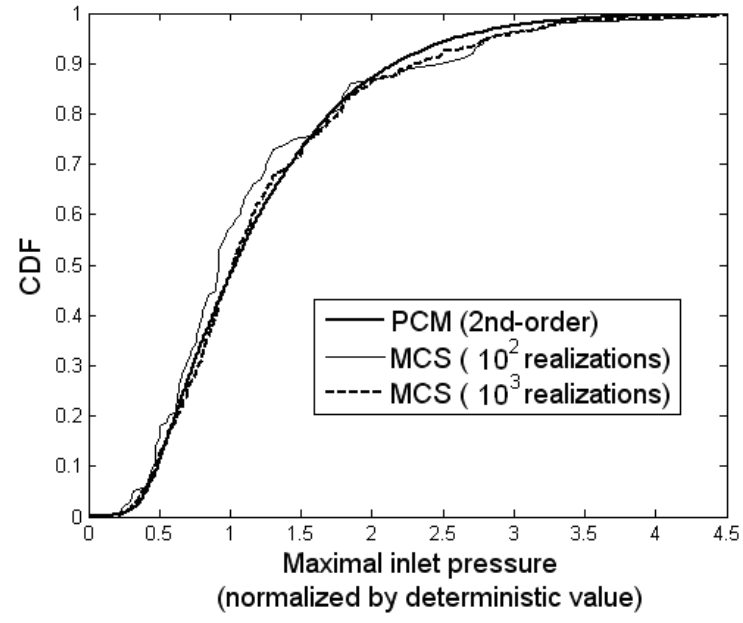

(b)

Fig. 3 Convergence of statistics of maximal inlet pressure:

(a) Mean (normalized by deterministic value); (b) CDF. 


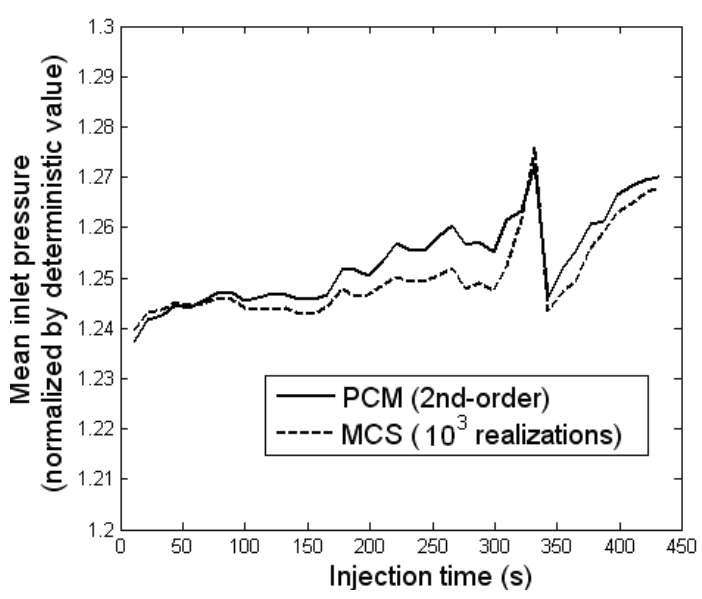

(a)

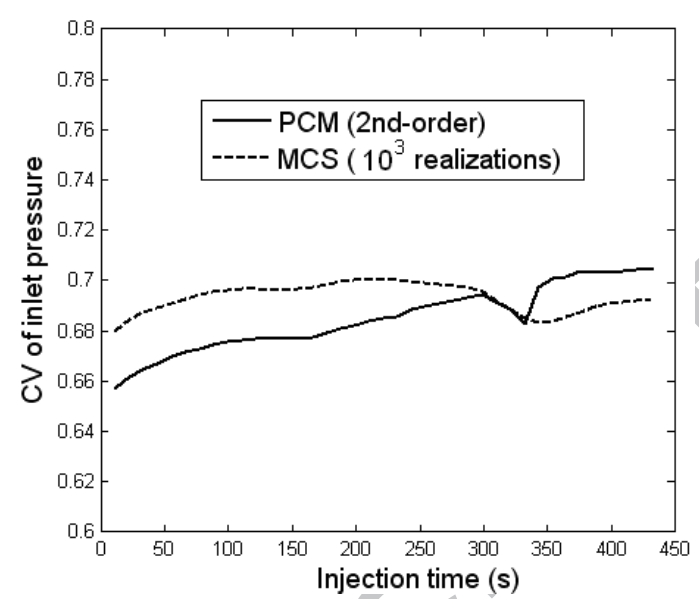

(b)

Fig. 4 Time history of statistical moments of inlet pressure:

(a) Mean (normalized by deterministic value); (b) CV.

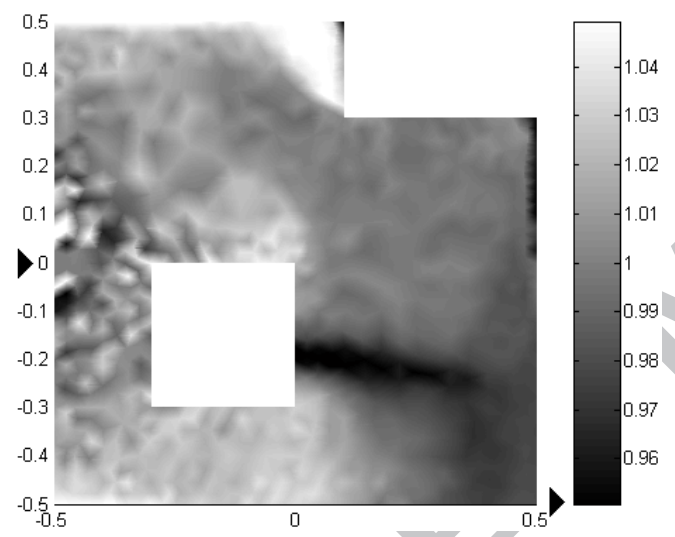

(a)

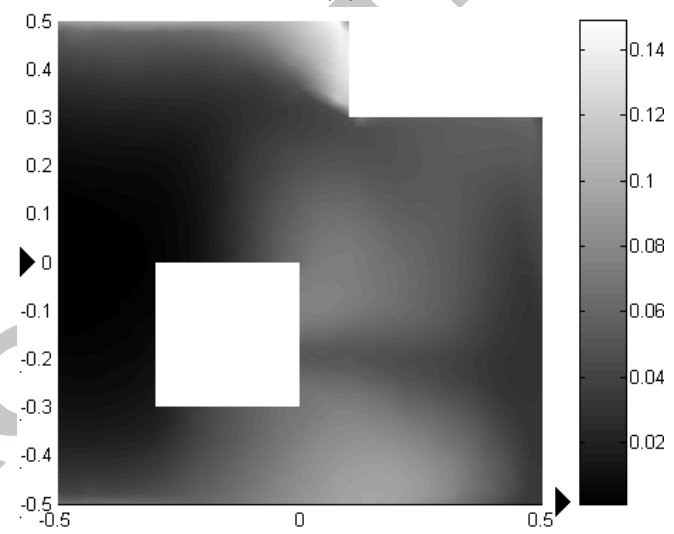

(c)

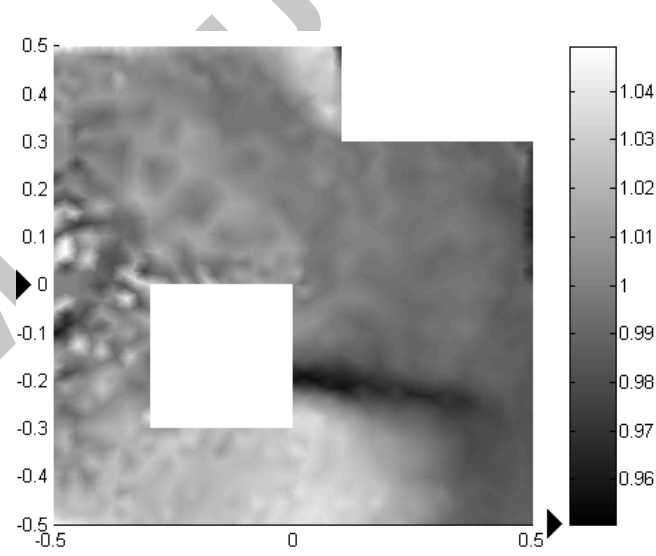

(b)

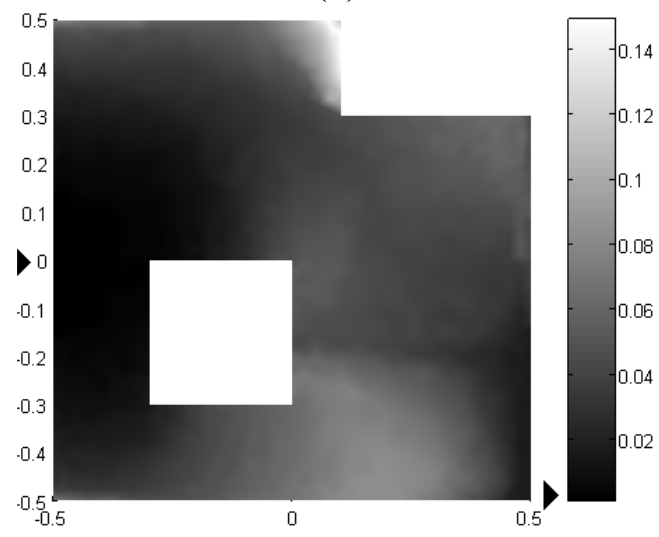

(d)

Fig. 5 Statistical moments of fill time (constant flow rate injection) comparing between MCS $\left(10^{3}\right.$ realizations) and PCM ( $2^{\text {nd }}$-order $)$ estimation:

(a) MCS estimation of mean value (normalized by deterministic value);

(b) PCM estimation of mean value (normalized by deterministic value);

(c) MCS estimation of CV; (d) PCM estimation of CV. 


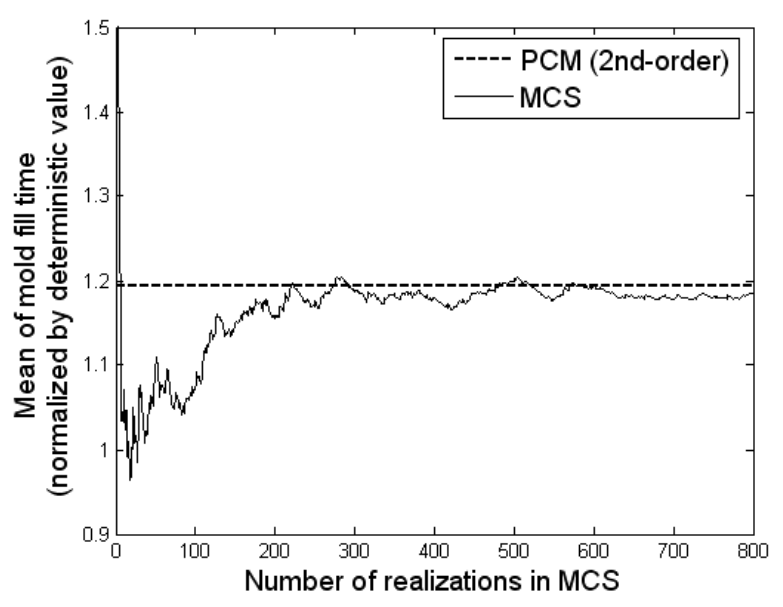

(a)

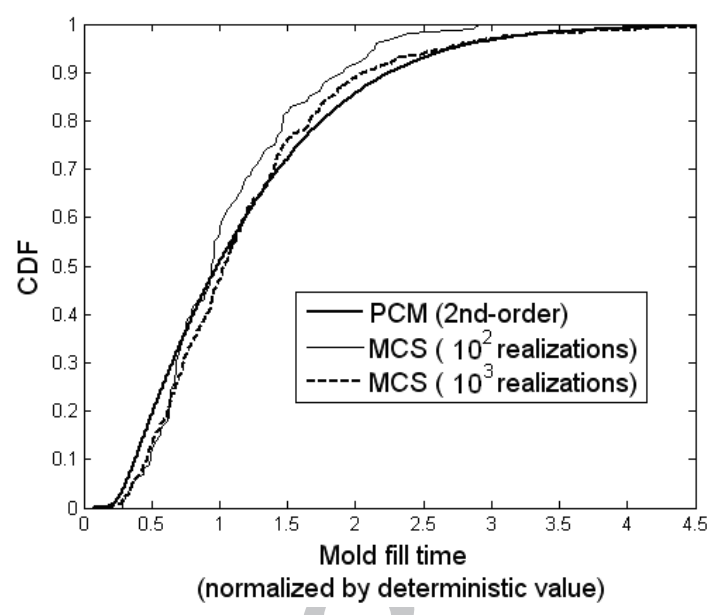

(b)

Fig. 6 Convergence of statistics of mold fill time (constant pressure injection):

(a) Mean (normalized by deterministic value); (b) CDF.

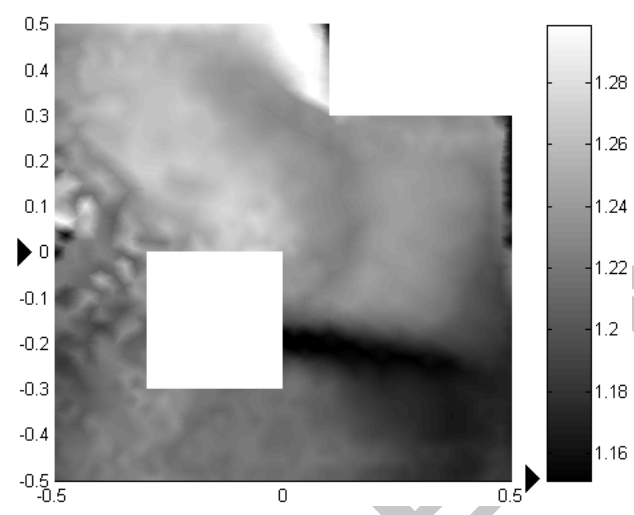

(a)

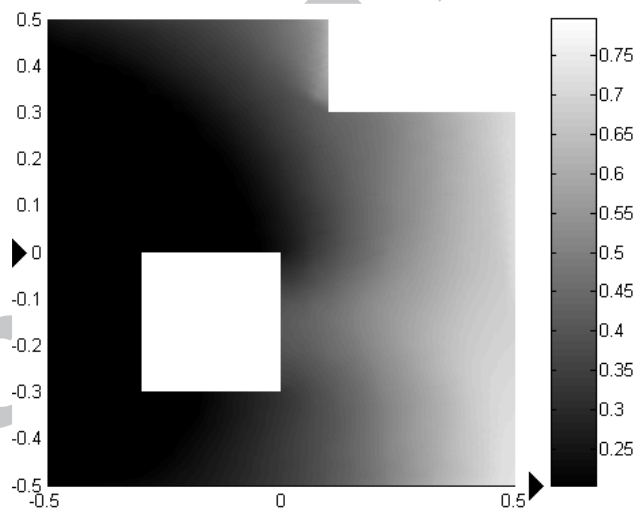

(c)

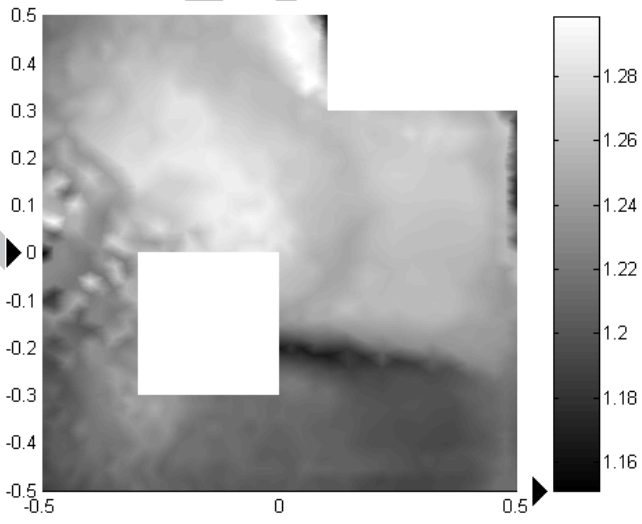

(b)

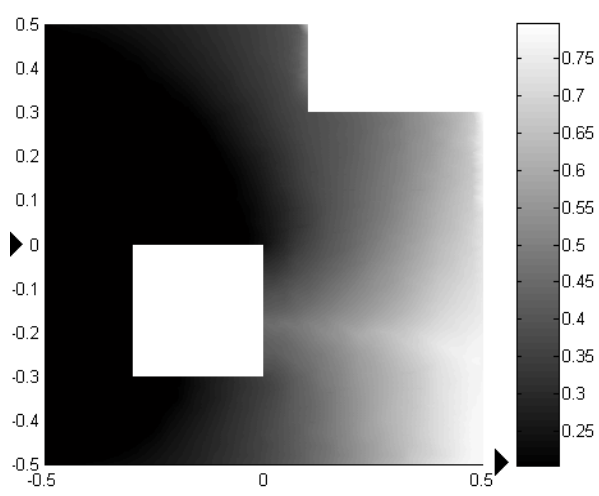

(d)

Fig. 7 Statistical moments of fill time (constant pressure injection) comparing between MCS ( $10^{3}$ realizations) and PCM $\left(2^{\text {nd }}\right.$-order $)$ estimation:

(a) MCS estimation of mean value (normalized by deterministic value);

(b) PCM estimation of mean value (normalized by deterministic value);

(c) MCS estimation of CV; (d) PCM estimation of CV. 
- Efficient numerical simulation of mold filling with random permeability as input

- Use of dimension-reduction techniques to represent random fields

- Coupling of Level Set Method and Probabilistic Collocation Method

- Numerical examples for injection schemes compared to Monte Carlo Simulation 